\title{
Assessment of the Association of HIV Infection with Hepatic Steatosis or Fibrosis: a Cross- Sectional, Case-Control Study
}

\author{
Paula Debroy \\ Jacobi Medical Center
}

Sanjana Nagraj ( $\square$ nagrajs@nychhc.org )

Jacobi Medical Center https://orcid.org/0000-0002-6230-7214

Natalia Chamorro-Pareja

Jacobi Medical Center

Neva Castro

Stony Brook University Medical Center: Stony Brook University Hospital

Alejandro de Leon

Baylor College of Medicine

Arcelia Guerson-Gil

Jacobi Medical Center

Orlando Quintero

Jacobi Medical Center

Isaac Laniado

Jacobi Medical Center

Michael Kladas

North Central Bronx Hospital

Dimpi Desai

Jacobi Medical Center

Priyanka Mathias

Jacobi Medical Center

Jason Leider

Jacobi Medical Center

Preeti Kishore

Jacobi Medical Center

Leonidas Palaiodimos

Jacobi Medical Center

Donald P Kotler

Jacobi Medical Center 


\section{Research Article}

Keywords: Steatosis, Fibrosis, HIV, NAFLD, liver, obesity, diabetes, HIS, FIB-4

Posted Date: June 1st, 2021

DOl: https://doi.org/10.21203/rs.3.rs-237921/v1

License: (c) (i) This work is licensed under a Creative Commons Attribution 4.0 International License. Read Full License

Version of Record: A version of this preprint was published at SN Comprehensive Clinical Medicine on September 23rd, 2021. See the published version at https://doi.org/10.1007/s42399-021-01054-4. 


\section{Abstract}

Background: Human immunodeficiency virus (HIV) infection and antiretroviral therapy have been associated with non-alcoholic fatty liver disease (NAFLD), but few studies have evaluated whether HIV infection is an independent risk factor for the development of hepatic steatosis and advanced liver fibrosis.

Objectives: To study the prevalence and severity of hepatic steatosis and advanced fibrosis in people living with HIV and control outpatients.

Methods: We conducted a cross-sectional analysis of relevant data from 875 pairs of individuals belonging to an HIV-dedicated outpatient clinic and an adult primary care clinic of an inner-city hospital. Hepatic Steatosis Index (HSI) and FIB-4 index were calculated as non-invasive measures of steatosis and fibrosis, respectively. A multivariate logistic regression analysis was performed to assess predictors of steatosis and advanced fibrosis.

Results: The prevalence of hepatic steatosis, determined by HSI $\geq 36$, was higher in HIV-negative subjects ( $71.5 \%$ vs. $65.4 \%, p=0.006$ ). The prevalence of advanced fibrosis, determined by FIB- 4 index $\geq 3.25$, was higher in the HIV-positive group ( $7 \%$ vs. $1.7 \%, p<0.001)$. Multivariable analysis did not identify HIV infection to be an independent risk factor for hepatic steatosis $(p=0.068)$ and advanced fibrosis.

Conclusions: In this cohort, hepatic steatosis was more prevalent in non-HIV infected patients, while advanced fibrosis had a higher prevalence in people living with HIV. HIV infection was not found to be an independent risk factor for either hepatic steatosis or fibrosis.

\section{Introduction}

Hepatic steatosis is common in HIV infection and is associated with poor outcomes and increased mortality [1]. In the pre-antiretroviral therapy (ART) era, steatosis was the most common cause of abnormal liver function tests [2]. In the early ART era, hepatic steatosis was mainly related to the lipodystrophy syndrome with an increase in visceral fat content, and medication toxicity seen with thiamine analog nucleoside reverse transcriptase inhibitors (NRTIs) and specific protease inhibitors [3]. Advances in drug development have decreased the metabolic toxicity of antiretroviral therapy. Currently, it is uncertain whether chronic HIV infection confers an increased risk of hepatic steatosis. 
Data on the prevalence and consequences of non-alcoholic fatty liver disease (NAFLD) in people living with HIV and on the risk factors for progression to fibrosis are limited. The reported prevalence of steatosis in HIV infection varies from 20-63\% [2-4]. There is controversy over the prevalence compared to the general population, and only a few studies have compared HIV-infected and uninfected adults [5, 6]. Obesity contributes to the development of steatosis, though most studies of NAFLD in people living with HIV do not stress this factor [5-14]. The Bronx has a high prevalence of obesity and diabetes when compared to the other New York boroughs, both of which are well-known risk factors for hepatic steatosis [15-17].

The objective of this study was to assess the association of HIV infection with hepatic steatosis by comparing the prevalence of hepatic steatosis in people living with HIV and non-HIV individuals attending outpatient clinics at an inner-city hospital in the Bronx, New York.

\section{Methods}

\section{Study population}

This was a cross-sectional study of 875 adults living with HIV infection who were followed in the HIVdedicated outpatient clinic at Jacobi Medical Center, Bronx, NY from January 1 through December 31, 2014. Controls included adult patients who have been tested negative for HIV and were followed in the adult primary care clinic over the same period. The Institutional Review Board at the Albert Einstein College of Medicine and the respective committee of NYC Health + Hospitals approved this study. The study was conducted according to STROBE guidelines for reporting observational studies [18]. The STROBE statement checklist is presented in the supplementary material.

\section{Data collection}

Clinical and laboratory data were obtained from the electronic medical record. The following variables were collected: age; gender; weight; height; ethnicity; self-reported tobacco and alcohol use, history of diabetes [defined as ongoing use of anti-diabetic medications or hemoglobin A1c ( $\mathrm{Hgb} \mathrm{A1c}$ ) greater or equal to $6.5 \%$ ], hypertension (defined as ongoing use of antihypertensive medication or documented under the problem list of the electronic medical record), and dyslipidemia (defined as current use of any lipid-lowering medication or documentation in the problem list of the electronic medical record). The diagnosis of prediabetes was defined using established American Diabetes Association (ADA) Hgb A1c criteria (5.7\%-6.4\%), fasting blood glucose $100-126 \mathrm{mg} / \mathrm{dL}$, or a two-hour serum glucose $140-200$ $\mathrm{mg} / \mathrm{dL}$ following an oral $75 \mathrm{gm}$ glucose tolerance test on patients that did not have a pre-existing diagnosis of diabetes [19]. HIV-specific data included current use of ART, duration of ART use, and years since diagnosis. Virologic suppression was defined as HIV RNA viral load $<20 / \mathrm{ml}$. Body mass index (BMI) was defined as weight divided by the height in meters squared $\left(\mathrm{kg} / \mathrm{m}^{2}\right)$. Baseline laboratory investigations included aspartate aminotransferase (AST), alanine aminotransferase (ALT), total cholesterol, low-density lipoprotein cholesterol (LDL), high-density lipoprotein cholesterol (HDL), triglyceride, Hgb A1c, platelet count, CD4 T lymphocyte (CD4) count, and HIV-1 RNA (copies/ml). Hepatitis 
C serological status was determined by antibody testing (anti-HCV antibody). Hepatitis B serological status was determined by surface antigen testing (HBsAg).

\section{Liver steatosis and fibrosis assessment}

Hepatic Steatosis Index (HSI) and FIB-4 index were calculated for all patients as they are non-invasive, inexpensive, and readily available methods that assess steatosis and fibrosis, respectively and can be used as screening tools in inner-city HIV clinics [20]. HSI was quantified by the formula HSI $=8 \times \mathrm{ALT} / \mathrm{AST}$ ratio + $\mathrm{BMI}(+2$, if history of diabetes; +2 , if female). A HIS $<30$ has been found to exclude NAFLD with a sensitivity of $93.1 \%$ and hold a negative predictive value of $85.2 \%$, while $\mathrm{HSI}>36$ has been shown to detect NAFLD with a specificity of $93.1 \%$ and have a positive predictive value of $86.7 \%$ [21]. Steatosis was defined as $\mathrm{HSI}>36$. HSI has also been validated in HIV mono-infected patients with an AUROC of 0.88 and an accuracy of $84.5 \%$ when compared to ultrasound and cross-validated against ${ }^{1} \mathrm{H}$ magnetic resonance spectroscopy $[20,21]$.

Advanced fibrosis was defined as a FIB-4 index higher than 3.25. FIB-4 index was calculated using the formula FIB-4 $=\left[\right.$ Age (years) $\times$ AST (IU/L)] / [Platelets $\left(10^{9} / \mathrm{L}\right) \times \sqrt{ }$ ALT (IU/L)]. A FIB-4 index of lower than 1.45 has a negative predictive value of $94.7 \%$ to exclude a significant fibrosis (METAVIR F3-F4) with a sensitivity of $74.3 \%$, while a FIB-4 higher than 3.25 offers a positive predictive value of $82.1 \%$ to identify extensive fibrosis (METAVIR F3-F4) with a specificity of 98.2\% [22].

\section{Statistical analysis}

Variables are expressed as median and interquartile range (IQR) for continuous and percentage for categorical variables. Comparisons between continuous variables were performed using the MannWhitney U test and between nominal variables using the Pearson's chi-squared test. Alpha value of statistical significance was set at 0.05 . Multivariate logistic regression analysis was performed to assess predictors of steatosis with his $\geq 36$ or advanced fibrosis. Only significant variables $(p<0.05)$ were then analyzed in a stepwise forward multivariate analysis model. Variables that were included in the FIB-4 and $\mathrm{HSI}$ equation were excluded from the analysis. HCV viral load was not included in the analysis due to the extent of missing data, particularly in the HIV-negative cohort. Odds Ratios (OR) and 95\% confidence intervals $(\mathrm{Cl})$ were reported for $p$ values $<0.05$. Data was analyzed using the statistical software SPSS (version 23.0, IBM, New York, NY, USA).

\section{Results}

\section{Baseline Characteristics}

A total of 1750 (patients living with HIV 875) patient charts were reviewed. Baseline characteristics are presented in Table 1. The median patient age (IQR) was 51.7 years (41.5-58.8); $57 \%$ were female. The majority of patients were non-Caucasian. Patients living with HIV had higher rates of tobacco use (15.5\% vs $33.9 \%$, $p$ < 0.001$)$. Hepatitis $\mathrm{C}$ seropositivity was documented in $142 \mathrm{HIV}$-infected patients compared to 
24 HIV-uninfected patients $(p<0.001)$. Among the HIV-infected cases, $94 \%$ were on ART and $72 \%$ were virologically suppressed. The median (IQR) duration of antiretroviral therapy was 9 years $(6-15)$. 
Table 1

General characteristics of the study population a

\begin{tabular}{|c|c|c|c|}
\hline & $\begin{array}{l}\text { HIV negative } \\
(\mathrm{N}=875)\end{array}$ & $\begin{array}{l}\text { HIV positive } \\
(\mathrm{N}=875)\end{array}$ & P-value \\
\hline Age (years), median (IQR) & $52.1(41.4-60.1)$ & $51.1(41.8-58.8)$ & 0.254 \\
\hline Sex Male, $(\%)$ & $371(42.4)$ & $371(42.4)$ & NA \\
\hline Race, $(\%)$ & & & $<0.001$ \\
\hline Black & $106(12.1)$ & $427(48.8)$ & \\
\hline Hispanic & $265(30.3)$ & $300(34.3)$ & \\
\hline Caucasian & $23(2.6)$ & $53(6.1)$ & \\
\hline Other & $56(6.4)$ & $88(10)$ & \\
\hline Unknown & $425(48.6)$ & $7(0.8)$ & \\
\hline $\mathrm{BMI}\left(\mathrm{kg} / \mathrm{m}^{2}\right)$, median (IQR) & $29.1(25.7-34)$ & $27.1(23.6-32)$ & $<0.001$ \\
\hline Normal, (\%) & $178(20.5)$ & $292(33.8)$ & $<0.001$ \\
\hline Overweight, (\%) & $299(34.4)$ & 276 (31.9) & \\
\hline Obese, (\%) & $311(35.8)$ & $237(27.4)$ & \\
\hline Morbidly Obese, (\%) & $81(9.3)$ & $60(6.9)$ & \\
\hline Smoking, (\%) & $134(15.5)$ & 296 (33.9) & $<0.001$ \\
\hline Alcohol abuse, (\%) & $93(12.1)$ & $91(10.3)$ & 0.252 \\
\hline Hepatitis B, (\%) & $9(1.5)$ & $38(4.4)$ & 0.002 \\
\hline Hepatitis C, (\%) & $24(3.9)$ & $142(16.3)$ & $<0.001$ \\
\hline Undetectable HCV viral load (\%) & $47(33)$ & $9(60)$ & 0.045 \\
\hline ART, (\%) & & $819(94.1)$ & \\
\hline Viral suppression, (\%) & & $633(72.4)$ & \\
\hline Hepatic steatosis (HSI > 36), (\%) & $626(71.5)$ & $572(65.4)$ & $<0.001$ \\
\hline Advanced Fibrosis (FIB $4>3.25$ ), (\%) & $14(1.7)$ & $61(7)$ & $<0.001$ \\
\hline Hypertension, (\%) & $356(40.7)$ & $301(34.4)$ & 0.007 \\
\hline Hyperlipidemia, (\%) & $160(18.3)$ & $223(25.5)$ & $<0.001$ \\
\hline Diabetes, (\%) & $277(31.7)$ & $135(15.4)$ & $<0.001$ \\
\hline Pre-diabetes, (\%) & 207 (23.7) & $219(25)$ & 0.504 \\
\hline
\end{tabular}




\begin{tabular}{|c|c|c|c|}
\hline & $\begin{array}{l}\text { HIV negative } \\
(\mathrm{N}=875)\end{array}$ & $\begin{array}{l}\text { HIV positive } \\
(\mathrm{N}=875)\end{array}$ & P-value \\
\hline HSI, median (IQR) & $41.0(37.3-46.57)$ & $38.7(34.4-43.9)$ & $<0.001$ \\
\hline Hgb a1c (\%), median (IQR) & $5.7(5.4-6.5)$ & $5.5(5.2-5.9)$ & $<0.001$ \\
\hline Cholesterol (mg/dl), median (IQR) & $179(153-209)$ & $171(145-199)$ & $<0.001$ \\
\hline Triglycerides (mg/dl), median (IQR) & $113(79.5-171.5)$ & $123(84-184)$ & 0.022 \\
\hline $\mathrm{HDL}(\mathrm{mg} / \mathrm{dl})$, median (IQR) & $49(42-58)$ & $47(38-56)$ & $<0.001$ \\
\hline LDL (mg/dl), median (IQR) & $102(80.5-126.5)$ & $94(72-117)$ & $<0.001$ \\
\hline Fib 4, median (IQR) & $1.05(0.72-1.48)$ & $1.23(0.86-1.82)$ & $<0.001$ \\
\hline AST (U/I), median (IQR) & $24(20-29.5)$ & $26(22-34)$ & $<0.001$ \\
\hline ALT (U/I), median (IQR) & $22(17-32)$ & $23(17-33)$ & 0.903 \\
\hline CD4 count (cells/ $\mu \mathrm{l})$, median (IQR) & & $586(382-819)$ & NA \\
\hline HIV RNA viral load (copies/ml), median (IQR) & & $0(0-29,000)$ & NA \\
\hline \multicolumn{4}{|c|}{$\begin{array}{l}\text { Abbreviations: BMI, body mass index; IQR, interquartile range; } H S I \text {, hepatic steatosis index; AST, } \\
\text { aspartate aminotransferase; ALT, alanine aminotransferace; HIV, human immunodeficiency virus; ART, } \\
\text { anti-retroviral therapy; } \mathrm{HDL} \text {, high density lipoprotein; } L D L \text {, low density lipoprotein; } N A \text {, not applicable } \\
\text { Missing values: } B M I=14 \text {, cholesterol }=46, H D L=46, L D L=69 \text {, triglycerides }=46 \text {, alcohol abuse }=105 \text {, } \\
\text { hepatitis B serology }=283 \text {, hepatitis } C \text { serology }=254 .\end{array}$} \\
\hline
\end{tabular}

The median BMI for both cohorts was $29.1 \mathrm{~kg} / \mathrm{m}^{2}$ (IQR 24.7-34) in the HIV-negative group vs 27.1 $\mathrm{kg} / \mathrm{m}^{2}$ (IQR 23.6-32) in the HIV cohort. A total of 141 (8.1\%) individuals of the total cohort had class III obesity $\left(\mathrm{BMI} \geq 40 \mathrm{~kg} / \mathrm{m}^{2}\right)$ with a significantly higher percentage in the HIV-negative group $(9.3 \% \mathrm{vs} 6.9 \%$, $\mathrm{p}<0.005)$.

The prevalence of diabetes mellitus was significantly higher in the HIV-negative group ( $31.7 \%$ vs $15.4 \%, p$ $<0.001)$. There was no difference in the prevalence of pre-diabetes defined by Hgb A1c criteria ( $23.7 \%$ vs $25 \%, p=0.504$ ). The HIV-negative group had higher median Hgb A1c values, higher LDL levels, higher total cholesterol levels, and lower triglyceride levels.

\section{Hepatic Steatosis}

The prevalence of hepatic steatosis, defined as $\mathrm{HSI}>36$, was $68 \%$ in the entire cohort and was higher in HIV-negative individuals $(71.5 \%$ vs $65.4 \%, p=0.006)$. Self-reported alcohol use did not differ between the two groups. In the multivariable analysis, presence of HIV infection was not found to be an independent risk factor for the presence of hepatic steatosis $(p=0.068)$ (Table 2). Factors that were significantly associated with higher odds of steatosis were a diagnosis of hypertension and higher Hgb A1c levels. Hepatitis C infection had a lower odds ratio for steatosis, as did tobacco use. Because of incorporation of 
body mass index and the diagnosis of diabetes in the calculation of $\mathrm{HSI}$, these variables were excluded from the logistic regression analysis.

Table 2

Multivariate analysis of independent variables associated with hepatic steatosis

\begin{tabular}{|llll|}
\hline & Odds Ratio & $95 \% \mathrm{Cl}$ & P Value \\
\hline HIV & - & - & 0.068 \\
\hline Hypertension & 1.5 & $1.16-1.95$ & 0.002 \\
\hline Tobacco use & 0.59 & $0.46-0.77$ & $<0.001$ \\
\hline Cholesterol & - & - & 0.113 \\
\hline LDL & - & - & 0.051 \\
\hline Hgb A1c & 1.20 & $1.08-1.34$ & 0.001 \\
\hline AST & - & - & 0.101 \\
\hline HBV serostatus & - & - & 0.918 \\
\hline HCV serostatus & 0.58 & $0.41-0.83$ & 0.003 \\
\hline $\begin{array}{l}\text { N = 1198 individuals with steatosis. Abbreviations: Cl, confidence interval; HIV, human } \\
\text { immunodeficiency virus; Hgb a1c, hemoglobin a1c; AST aspartate aminotransferase; HBV, hepatitis B } \\
\text { virus; HCV, hepatitis C virus. }\end{array}$ & \\
\hline
\end{tabular}

\section{Advanced fibrosis}

The overall prevalence of advanced fibrosis, defined as FIB-4 index $>3.25$, was $4.4 \%$ and was higher in people living with HIV $(7 \%$ vs. $1.7 \%, p<0.001)$. In the multivariate analysis, independent factors associated with higher odds of advanced fibrosis were age $(\mathrm{OR}=1.06, \mathrm{Cl}=1.02-1.09, \mathrm{p}<0.001), \mathrm{HBV}$ infection $(\mathrm{OR}=2.82, \mathrm{Cl}=1.02-7.85, \mathrm{p}=0.05)$, and HCV infection $(\mathrm{OR}=8.35, \mathrm{Cl}=4.80-14.54, \mathrm{p}<0.001)$ (Table 3). HIV was not an independent risk factor for diagnosis of advanced fibrosis. The results of a multivariate analysis were similar when FIB-4 results were expressed as absolute values. Because of incorporation of ALT and AST in the FIB-4 index calculation, these variables were excluded from the logistic regression analysis. 
Table 3

Multivariate analysis variables associated with hepatic fibrosis

\begin{tabular}{|llll|}
\hline & Odds Ratio & $95 \% \mathrm{Cl}$ & P Value \\
\hline HIV & - & - & 0.226 \\
\hline Age & 1.05 & $1.02-1.08$ & $<0.001$ \\
\hline Gender & - & - & 0.719 \\
\hline Tobacco use & - & - & 0.693 \\
\hline BMI per 1 kg/m ${ }^{2}$ increment & 0.94 & $0.9-0.99$ & 0.018 \\
\hline Cholesterol & - & - & 0.657 \\
\hline LDL cholesterol & 0.99 & $0.98-0.99$ & 0.015 \\
\hline HDL cholesterol & - & - & 0.540 \\
\hline HBV serostatus & 2.82 & $1.02-7.85$ & 0.05 \\
\hline HCV serostatus & 8.35 & $4.80-14.54$ & $<0.001$ \\
\hline $\begin{array}{l}\mathrm{N}=75 \text { individuals with fibrosis. Abbreviations: Cl, confidence interval; HIV, human immunodeficiency } \\
\text { virus; BMl, body mass index; HBV, hepatitis B virus; HCV, hepatitis C virus }\end{array}$ & \\
\hline
\end{tabular}

\section{Discussion}

In this cohort of patients living with HIV, we found a lower prevalence of hepatic steatosis compared to the control cohort of individuals without HIV despite a higher prevalence of HCV seropositivity. The prevalence of hepatic steatosis in both cohorts was higher than anticipated (>50\% for both groups). Notably, HIV was not associated with an increased risk of steatosis or fibrosis in this study.

The overall prevalence of hepatic steatosis in patients with HIV in our cohort was $65.4 \%$, a finding that was higher than other studies using imaging and invasive studies as diagnostic tools $[4,7]$. There are several factors that could potentially account for the higher prevalence rate of hepatic steatosis in our study. The median BMI for HIV positive cases with steatosis was $30.2 \mathrm{~kg} / \mathrm{m}^{2}$, studies with lower prevalence rates of fatty liver had a mean BMI in the normal category $[5,10]$, while studies showing higher prevalence rates of more than $50 \%$, had mean BMls in the overweight category [7]. Also, our study did not exclude patients with alcohol use or hepatitis $C$. The rate of self-reported alcohol use was not statistically different between both groups. Hepatitis $\mathrm{C}$ was found not to be associated with hepatic steatosis in the multivariate regression analysis.

While BMI is known to explain much of the variance in $\mathrm{HSI}$, it is not the most accurate predictor of hepatic adiposity, as opposed to waist circumference or visceral fat content, both of which more closely reflect the presence of metabolic syndrome [23]. A prior study of liver biopsies in patients undergoing bariatric 
surgery showed that the presence of steatosis was significantly related to measures of insulin resistance rather than to BMI [24]. Also, hepatic fat content, as estimated by proton spectroscopy, was a stronger predictor of insulin resistance by the Homeostasis Model Assessment (HOMA) than was visceral fat content, as determined by magnetic resonance imaging [25].

The prevalence of advanced fibrosis determined by FIB $4>3.25$ in our HIV cohort is similar to a recent meta-analysis where the pooled prevalence was $21 \%$ with the highest reported prevalence of $35 \%[2,12]$. The prevalence of hepatic fibrosis in our study was higher in the HIV cohort compared to non-HIV individuals. The main factor associated with the increased prevalence of advanced fibrosis in HIV positive cases was a higher rate of HCV infection. HIV itself was not related with an increased risk of advanced fibrosis in our study. HCV infection in HIV-positive individuals has been shown to be associated with an increased risk of progression to fibrosis [26].

Our findings of lower prevalence rates of hepatic steatosis in patients living with HIV is compatible with the only other large study done with HIV-negative controls [5]. This may be in part due to currently used ART, which unlike the previously used agents, is not implicated in medication-induced hepatotoxicity and does not serve as a risk factor for increased incidence of steatosis in HIV positive patients. In fact, data suggest that cumulative exposure to ART reduces the risk of developing hepatic steatosis $[5,27]$.

Cross-sectional studies in HIV positive patients have looked at the prevalence of steatosis determined by non-invasive methods, including ultrasound, CT scan, and elastography, with prevalence ranging from 13 to $55 \%$ [2-4]. Studies including HCV co-infected patients have similar prevalence rates $[5,28,29]$. These radiological techniques can be expensive and are not readily available to inner-city HIV clinics in lowincome settings. There are limited liver biopsy assessment studies and they often include high-risk patients with advanced disease or unexplained elevation of transaminases $[7,11]$. These diagnostic modalities are also unlikely to be readily available in low-income settings. Several non-invasive serological methods for the diagnosis of hepatic steatosis have been validated in HIV positive patients, including the liver fat score (LFS), the lipid accumulation product, and HIS [20,30]. Previous studies with serological and diagnostic modalities were found to significantly underestimate the prevalence of NAFLD [31], but the high prevalence of steatosis in our study with the use of HSI is similar to other prevalence studies which suggests that it is adequate for use in HIV clinics where metabolic abnormalities are prevalent.

We acknowledge the strengths and limitations of our study. This is the largest study conducted to date comparing the prevalence of hepatic steatosis in people living with HIV and HIV-negative controls. This study used accessible and noninvasive clinical markers to estimate hepatic steatosis so that these markers can be easily incorporated during evaluation of patients in HIV clinics. However, we recognize that the estimation of hepatic steatosis and fibrosis were based on surrogate markers. The HSI calculation includes BMI and the diagnosis of diabetes, both of which were common in our cohort. While transient elastography and other sophisticated measurements may be more sensitive tools to estimate hepatic steatosis in both HIV-negative and positive individuals they were not available at our institution at 
the time of data collection [28, 32]. Information on the use of thymidine analog NRTIs or specific Pls was not recorded. HCV and HBV viral loads were not available for many patients and treatment status was uncertain. Finally, HgbA1c testing may underestimate serum glucose in HIV-infected patients with reduced red cell survival [33].

\section{Conclusions}

In summary, while hepatic steatosis was more prevalent in non-HIV infected patients and advanced fibrosis had a higher prevalence in the HIV-positive cohort, HIV infection itself did not seem to significantly influence the development of either steatosis or fibrosis. The ability of non-invasive clinical predictors to guide therapy and improve clinical outcomes remains to be determined.

\section{Declarations}

Funding Dr. Donald Kotler has received funding for work outside of the submitted manuscript: from Abbvie, for research grants. None of the other authors have any relationships to disclose.

Conflicts of interest: On behalf of all authors, the corresponding author states that there is no conflict of interest.

Ethics approval This project was approved by the Albert Einstein College of Medicine Institutional Review Board. A consent waiver was provided.

Consent to participate Not applicable

Consent for publication Not applicable

Availability of data and material The dataset can be provided after data use agreement among Jacobi Medical Center and the requester's institution is requested and approved.

Code availability Not applicable

Authors' contributions Drs. Debroy, Leider, and Kotler contributed to the conception of this project. Drs. Nagraj, Chamorro-Pareja, Palaiodimos, and de Leon designed the study. Drs. Debroy, Nagraj, ChamorroPareja, Palaiodimos, Castro, Quintero, Mathias, Laniado, Guerson-Gil, Kladas, and Desai performed the data collection. Drs. Palaiodimos, Leider, and Kotler performed the statistical analysis. All authors evaluated the results of the analysis and substantially contributed to the interpretation of results and drafting of the article. Drs. Debroy, Kotler, Nagraj, Chamorro Pareja, and Palaiodimos critically revised the manuscript. All authors approved the final version.

\section{References}


1. Palella, F.J., Jr., et al., Mortality in the highly active antiretroviral therapy era: changing causes of death and disease in the HIV outpatient study. J Acquir Immune Defic Syndr, 2006. 43(1): p. 27-34.

2. Maurice, J.B., et al., Prevalence and risk factors of nonalcoholic fatty liver disease in HIVmonoinfection. AIDS, 2017. 31(11).

3. Kapoor, N., et al., A gathering storm: HIV infection and nonalcoholic fatty liver disease in low and middle-income countries. Aids, 2019. 33(7): p. 1105-1115.

4. Lake, J.E., et al., Expert Panel Review on Non-Alcoholic Fatty Liver Disease in Persons With HIV. Clin Gastroenterol Hepatol, 2020.

5. Price, J.C., et al., Risk factors for fatty liver in the Multicenter AIDS Cohort Study. Am J Gastroenterol, 2014. 109(5): p. 695-704.

6. Mohammed, S.S., et al., HIV-positive patients with nonalcoholic fatty liver disease have a lower body mass index and are more physically active than HIV-negative patients. J Acquir Immune Defic Syndr, 2007. 45(4): p. 432-8.

7. Morse, C.G., et al., Nonalcoholic Steatohepatitis and Hepatic Fibrosis in HIV-1-Monoinfected Adults With Elevated Aminotransferase Levels on Antiretroviral Therapy. Clin Infect Dis, 2015. 60(10): p. 1569-78.

8. Crum-Cianflone, N., et al., Nonalcoholic fatty liver disease among HIV-infected persons. J Acquir Immune Defic Syndr, 2009. 50(5): p. 464-73.

9. Guaraldi, G., et al., Nonalcoholic fatty liver disease in HIV-infected patients referred to a metabolic clinic: prevalence, characteristics, and predictors. Clin Infect Dis, 2008. 47(2): p. 250-7.

10. Nishijima, T., et al., Traditional but not HIV-related factors are associated with nonalcoholic fatty liver disease in Asian patients with HIV-1 infection. PloS one, 2014. 9(1): p. e87596-e87596.

11. Sterling, R.K., P.G. Smith, and E.M. Brunt, Hepatic steatosis in human immunodeficiency virus: a prospective study in patients without viral hepatitis, diabetes, or alcohol abuse. J Clin Gastroenterol, 2013. 47(2): p. 182-7.

12. Vodkin, I., et al., Clinical, biochemical and histological differences between HIV-associated NAFLD and primary NAFLD: a case-control study. Aliment Pharmacol Ther, 2015. 41(4): p. 368-78.

13. Macías, J., et al., Prevalence and factors associated with liver steatosis as measured by transient elastography with controlled attenuation parameter in HIV-infected patients. Aids, 2014. 28(9): p. 1279-87.

14. Lombardi, R., et al., Liver test abnormalities in patients with HIV mono-infection: assessment with simple noninvasive fibrosis markers. Annals of gastroenterology, 2017. 30(3): p. 349-356.

15. Ong, J.P. and Z.M. Younossi, Epidemiology and natural history of NAFLD and NASH. Clin Liver Dis, 2007. 11(1): p. 1-16, vii.

16. Aguilar, E., Obesity and Diabetes. Wellness Newsletter, 2017.

17. Maurice, J.B., et al., Increased BMI and Type 2 diabetes are the main predictors of NAFLD and advanced fibrosis in liver biopsies of patients with HIV mono-infection. Clin Infect Dis, 2020. 
18. Observational studies: getting clear about transparency. PLoS Med, 2014. 11(8): p. e1001711.

19. 2. Classification and Diagnosis of Diabetes. Diabetes Care, 2017. 40(Supplement 1): p. S11-S24.

20. Sebastiani, G., et al., Incidence and predictors of hepatic steatosis and fibrosis by serum biomarkers in a large cohort of human immunodeficiency virus mono-infected patients. Open forum infectious diseases, 2015. 2(1): p. ofv015-ofv015.

21. Lee, J.H., et al., Hepatic steatosis index: a simple screening tool reflecting nonalcoholic fatty liver disease. Dig Liver Dis, 2010. 42(7): p. 503-8.

22. Vallet-Pichard, A., et al., FIB-4: an inexpensive and accurate marker of fibrosis in HCV infection. comparison with liver biopsy and fibrotest. Hepatology, 2007. 46(1): p. 32-6.

23. Després, J.P., et al., Abdominal obesity and the metabolic syndrome: contribution to global cardiometabolic risk. Arterioscler Thromb Vasc Biol, 2008. 28(6): p. 1039-49.

24. Gholam, P.M., et al., Nonalcoholic fatty liver disease in severely obese subjects. Am J Gastroenterol, 2007. 102(2): p. 399-408.

25. He, Q., et al., Insulin resistance, hepatic lipid and adipose tissue distribution in HIV-infected men. Antiviral therapy, 2008. 13(3): p. 423-428.

26. Sulkowski, M.S. and Y. Benhamou, Therapeutic issues in HIV/HCV-coinfected patients. Journal of Viral Hepatitis, 2007. 14(6): p. 371-386.

27. Woreta, T.A., et al., Incidence and risk factors for steatosis progression in adults coinfected with HIV and hepatitis C virus. Gastroenterology, 2011. 140(3): p. 809-817.

28. Macias, J., et al., Impact of genetic polymorphisms associated with nonalcoholic fatty liver disease on HIV-infected individuals. Aids, 2015. 29(15): p. 1927-35.

29. Li Vecchi, V., et al., Prospective evaluation of hepatic steatosis in HIV-infected patients with or without hepatitis C virus co-infection. Int J Infect Dis, 2012. 16(5): p. e397-402.

30. Siddiqui, M.S., et al., Validation of noninvasive methods for detecting hepatic steatosis in patients with human immunodeficiency virus infection. Clin Gastroenterol Hepatol, 2015. 13(2): p. 402-5.

31. Younossi, Z.M., et al., Global epidemiology of nonalcoholic fatty liver disease-Meta-analytic assessment of prevalence, incidence, and outcomes. Hepatology, 2016. 64(1): p. 73-84.

32. Ryan, P., et al., Predictors of severe hepatic steatosis using abdominal ultrasound in HIV-infected patients. HIV Med, 2009. 10(1): p. 53-9.

33. Kim, P.S., et al., A1C underestimates glycemia in HIV infection. Diabetes Care, 2009. 32(9): p. $1591-3$.

\section{Supplementary Files}

This is a list of supplementary files associated with this preprint. Click to download.

- STROBEchecklistcombinedPlosMedicine.docx 